H. E. Brink, Department of Mechanical Engineering, Technikon Free State, Private Bag X 20539, Bloemfontein, 9300, South Africa. e-mail:

hbrink@eng.tofs.ac.za

P. Maritz, Department of Mathematics, University of Stellenbosch, Private

Bag X1, Matieland, 7602, South Africa. e-mail: pm@maties.sun.ac.za

\title{
ON THE EXTENSION OF SET-VALUED SET FUNCTIONS
}

\begin{abstract}
We extend additive set-valued set functions and normal multimeasures defined on a ring of subsets. We also prove a Carathéodory-HahnKluvanek-type theorem for additive set-valued set functions. Finally, we establish results on the extension of transition multimeasures.
\end{abstract}

\section{Introduction}

The theory of set-valued measures (multimeasures) is a natural extension of the classical theory of vector measures, where the set function is allowed to become set-valued instead of single-valued. The study of multimeasures was first motivated by the needs of mathematical economics and, in particular, the search for equilibria in exchange economies with production, in which coalitions are the primary economic units (see [31] and [13]). From this point of departure, Godet-Thobie has developed the subject of multimeasures extensively during 1970 to 1975 in a series of papers ([16], [17], [18], [19], [20]) culminating in her Ph.D-thesis [21] in 1975. The subject of multimeasures has since then attracted the interest of many mathematicians, who contributed important results, both in theory and in applications, in the fields of control systems, statistics, mathematical economics, game theory, etc.

Loosely speaking, one calls $M$ a multimeasure if the range space $X$ is (at least) a commutative topological group and $M$ is suitably countably additive. Central to the approaches that have been taken appear to be the definitions of convergence of an infinite sum of subsets of $X$. Furthermore, different types of approaches can be distinguished according to the range space including the

Key Words: Extension, multimeasure

Mathematical Reviews subject classification: 28B20, 28B05, 46G10

Received by the editors February 1, 2000 
values of the multimeasures. Significant contributions to the study of multimeasures were made by Artstein [3], Debreu and Schmeidler [13], Schmeidler [30], Wenxiu, Jifeng and Aijie [33] for $\mathbb{R}^{n}$-valued multimeasures, by Aló, de Korvin and Roberts [1,2], Costé [6-9], Hiai [22], Papageorgiou [26-29] and Kandilakis [23] for Banach space-valued multimeasures and by Castaing [5], Costé and Pallu de la Barriére [10,11] and Godet-Thobie [19,21] for multimeasures with values in a locally convex vector space.

The extension problem for countably additive scalar measures has its roots in integration theory. To apply the Lebesgue construction it was necessary to extend scalar set functions, usually defined explicitly only on a ring, to the sigma-algebra of measurable sets. However, the extension problem for vector measures has had a more difficult development. The most inclusive statement about the extension theorem for vector measures has been given by Kluvanek [24]. On the other hand, the extension of set-valued set functions (and in particular additive set-valued set functions) has been neglected. While results on the extension of additive set-valued set functions are basically nonexistent, only two approaches on the extension of multimeasures were thus far established. In [23] Kandilakis considered the extension of Banach space-valued multimeasures while in [33] Wenxiu, Jifeng and Aijie proved extension results for multimeasures with values in a finite-dimensional space. In this paper it is our purpose to study the extension of additive set-valued set functions and multimeasures in general. We also establish results on the extension of transition multimeasures; i.e., multimeasures parametrized by the elements of a measurable space. Transition multimeasures are useful in the study of Markov temporary equilibrium processes in dynamic economies (see Blume [4]).

The organization of the paper is as follows.

We start by establishing the necessary notations and definitions that go along with the subject of multimeasures. In the second section we look at the extension of set-valued set functions defined on a ring of subsets. In particular, we establish results on the extension of additive set-valued set functions and normal multimeasures. In the last section our multimeasures are now defined on an algebra of subsets and are being extended to the generated sigma-algebra. In the process we will give a Carathéodory-Hahn-Kluvanektype theorem for additive set-valued set functions (see Theorem 2 on page 27 of [14]), thereby extending the corresponding result (Theorem 2.6) of Kandilakis [23] to additive set-valued set functions (see also Corollary 3 on page 28 of [14]). In the last section we extend transition multimeasures. 


\section{Preliminaries}

Let $T$ be a non-empty point set on which no topological structure is required and let $X$ be a linear topological space with topological dual $X^{\prime}$. We denote by $\mathcal{P}(X)$ the class of all nonempty subsets of $X$. Furthermore, by $\mathcal{P}_{f(b)}(X)$ (respectively, $\left.\mathcal{P}_{k}(X)\right)$ we will denote the closed (bounded) (respectively, compact) sets in $\mathcal{P}(X)$. A $c$ after $f(b)$ or $k$ will mean that the set is in addition convex. A $w$ in front of $f(b)$ (respectively, $k$ ) means that the closedness (respectively, compactness) is with respect to the weak topology $w\left(X, X^{\prime}\right)$.

We now let $(X, d)$ be a metric space. Then the distance between a point $x \in X$ and a non-empty set $A \subseteq X$ is defined as $d(x, A)=\inf \{d(x, a)$ $a \in A\}$. Furthermore, for any $A, B \in \mathcal{P}_{k}(X)$, we define their Hausdorff semimetric by $d(A, B)=\sup \{d(a, B) \mid a \in A\}$, and their Hausdorff metric by $H(A, B)=\max \{d(A, B), d(B, A)\}$. In addition, we put $\|A\|=H(A,\{0\})$ (the norm of the set $A$ ). Whenever we refer to the metric space $\mathcal{P}_{k}(X)$, it must be understood that $\mathcal{P}_{k}(X)$ is equipped with the Hausdorff metric $H$.

For $A \in \mathcal{P}(X)$, we let $\bar{A}$ denote the closure of $A$ and $\overline{c o} A$ denote the closed convex hull of $A$. For all $x^{\prime} \in X^{\prime}$, we set $\sigma\left(x^{\prime}, A\right)=\sup \left\{\left(x^{\prime}, x\right) \mid x \in A\right\}$ (the support function of $A$ ).

Unless otherwise specified we will assume that $\mathcal{A}$ is an arbitrary nonempty class of subsets of $T$. By a set-valued set function we mean a relation defined on $\mathcal{A}$ and with values in $\mathcal{P}(X)$. Furthermore, if $A, B \in \mathcal{P}(X)$, then we put

$$
A+B=\{a+b \mid a \in A, b \in B\} .
$$

Definition 2.1. If $X$ is a linear topological space, then a set-valued set function $M: \mathcal{A} \rightarrow \mathcal{P}(X)$ is said to be punctually additive if

$$
M(A \cup B)=M(A)+M(B)
$$

for every pair $A, B \in \mathcal{A}$ of disjoint sets such that $A \cup B \in \mathcal{A}$.

Definition 2.2. If $X$ is a linear topological space, then a set-valued set function $M: \mathcal{A} \rightarrow \mathcal{P}_{f}(X)$ is said to be additive if

$$
M(A \cup B)=\overline{M(A)+M(B)}
$$

for every pair $A, B \in \mathcal{A}$ of disjoint sets such that $A \cup B \in \mathcal{A}$.

As for single-valued set functions we have the concept of countable additivity: We say that a set-valued set function $M: \mathcal{A} \rightarrow \mathcal{P}(X)$ is countably additive if

$$
M\left(\bigcup_{k=1}^{\infty} A_{k}\right)=\sum_{k=1}^{\infty} M\left(A_{k}\right),
$$


for every sequence $\left(A_{k}\right) \subseteq \mathcal{A}$ of mutually disjoint sets such that $\cup_{k=1}^{\infty} A_{k} \in \mathcal{A}$. Depending on how we define the above infinite sum we obtain different notions of countably additivity, as will be seen below.

For the rest of this section we consider $(T, \mathcal{S})((\Omega, \mathcal{T})$, respectively), where $\mathcal{S}(\mathcal{T}$, respectively) is a $\sigma$-ring of subsets of $T(\Omega$, respectively). By a multimeasure we mean a countably additive set-valued set function $M: \mathcal{S} \rightarrow \mathcal{P}(X)$ such that $M(\emptyset)=\{0\}$. In particular, we will differentiate between the following types of multimeasures:

Definition 2.3. If $X$ is a linear topological space, then a set-valued set function $M: \mathcal{S} \rightarrow \mathcal{P}(X)$ is called a strong multimeasure if

(a) $M(\emptyset)=\{0\}$ and $M$ is punctually additive;

(b) for every $x_{k} \in M\left(A_{k}\right)$ the series $\sum_{k=1}^{\infty} x_{k}$ is unconditionally convergent and

$$
M\left(\bigcup_{k=1}^{\infty} A_{k}\right)=\left\{x \in X \mid x=\sum_{k=1}^{\infty} x_{k}, x_{k} \in M\left(A_{k}\right)\right\} .
$$

Definition 2.4. If $X$ is a linear topological space, then a set-valued set function $M: \mathcal{S} \rightarrow \mathcal{P}_{f}(X)$ is called a normal multimeasure if

(a) $M(\emptyset)=\{0\}$ and $M$ is additive;

(b) for every sequence $\left(A_{k}\right) \subseteq \mathcal{S}$ of mutually disjoint sets such that $A=$ $\cup_{k=1}^{\infty} A_{k}$, we have that

$$
\lim _{n \rightarrow \infty} H\left(M(A), \overline{\sum_{k=1}^{n} M\left(A_{k}\right)}\right)=0 .
$$

Definition 2.5. If $X$ is a linear topological space, then a set-valued set function $M: \mathcal{S} \rightarrow \mathcal{P}_{f}(X)$ is called a weak multimeasure if

(a) $M(\emptyset)=\{0\}$;

(b) for every $x^{\prime} \in X^{\prime}$ the set function $A \mapsto \sigma\left(x^{\prime}, M(A)\right)$ is a signed measure with values in $\mathbb{R} \cup\{+\infty\}$.

As for single-valued measures we have the notion of total variation of a set-valued set function. Let $X$ be a normed space and suppose that $M: \mathcal{A} \rightarrow$ $\mathcal{P}(X)$ is a set-valued set function such that $M(\emptyset)=\{0\}$ if $\emptyset \in \mathcal{A}$. For every $A \subseteq T$ we define the variation of $M$ on $A$, denoted by $v(M, A)$, by

$$
v(M, A)=\sup _{I} \sum_{i \in 1}\left\|M\left(A_{i}\right)\right\|
$$


where the supremum is taken for all the families $\left(A_{i}\right)_{i \in I} \subseteq \mathcal{A}$ of mutually disjoint sets contained in $A$. The set function $v(M)$ is called the variation of $M$ and the restriction of $v(M)$ to the class $\mathcal{A}$ will again be denoted by $v(M)$. We say that $M: \mathcal{A} \rightarrow \mathcal{P}(X)$ is of bounded variation (with respect to $\mathcal{A}$ ) if $v(M, A)<\infty$ for every $A \in \mathcal{A}$. If $M: \mathcal{S} \rightarrow \mathcal{P}(X)$ is a strong multimeasure, then the variation $v(M)$ of $M$ is a positive measure (see Proposition 1.1 on page 98 of [22]).

A set $A \in \mathcal{S}$ is said to be an atom for a multimeasure $M: \mathcal{S} \rightarrow \mathcal{P}(X)$ if $M(A) \neq\{0\}$ and if either $M(B)=\{0\}$ or $M(A \backslash B)=\{0\}$ holds for every $B \subseteq A, B \in \mathcal{S}$. We say that $M$ is atomic if there exists at least one atom in $\mathcal{S}$, and that $M$ is non-atomic if there are no atoms in $\mathcal{S}$.

If $\mu: \mathcal{S} \rightarrow X$ is a positive measure on $\mathcal{S}$, then we say that $M$ is $\mu$-continuous on $\mathcal{S}$ if and only if for any $A \in \mathcal{S}$ with $\mu(A)=0$ we have that $M(A)=\{0\}$. We call a measure $m: \mathcal{S} \rightarrow X$ a selector of $M$ if $m(A) \in M(A)$ for all $A \in \mathcal{S}$. We denote by $S_{M}$ the set of selectors of $M$. A set-valued set function $M: \mathcal{S} \rightarrow \mathcal{P}(X)$ is called strongly additive if for every sequence $\left(A_{k}\right) \subseteq \mathcal{S}$ of mutually disjoint sets the series $\sum_{k=1}^{\infty} x_{k}$ converges for any $x_{k} \in M\left(A_{k}\right)$, $k \in \mathbb{N}$.

Let $X$ be a separable Banach space and let $\mu$ be a positive measure on $\mathcal{S}$. A multifunction $F: T \rightarrow \mathcal{P}(X)$ is said to be $\mu$-measurable if and only if for every closed subset $C$ of $X$, the set $F^{-}(C)=\{t \in T \mid F(t) \cap C \neq \emptyset\}$ is $\mu$-measurable. A set-valued set function $M: \Omega \times \mathcal{S} \rightarrow \mathcal{P}_{f}(X)$ is said to be a transition multimeasure if and only if for all $A \in \mathcal{S}, \omega \mapsto M(\omega, A)$ is an $\mathcal{S}$-measurable multifunction and for all $\omega \in \Omega, A \mapsto M(\omega, A)$ is a multimeasure. We will distinguish between strong, normal and weak transition multimeasures. A selector transition measure (or simply a transition selector) of a transition multimeasure $M: \Omega \times \mathcal{S} \rightarrow \mathcal{P}_{f}(X)$ is a set function $m: \Omega \times \mathcal{S} \rightarrow X$ such that for all $A \in \mathcal{S}, \omega \mapsto m(\omega, A)$ is an $\mathcal{S}$-measurable function, for all $\omega \in \Omega$, $A \mapsto m(\omega, A)$ is a measure and for all $(\omega, A) \in \Omega \times \mathcal{S}, m(\omega, A) \in M(\omega, A)$. The set of all transition selectors of $M$ will be denoted by $T S_{M}$.

\section{Extension of Additive Set-Valued Set Functions}

We let $\mathcal{S}$ be a ring of subsets of $T$ and $\mu$ a positive, finite, subadditive and increasing set function on $\mathcal{S}$. Then the function $\rho_{\mu}: \mathcal{S} \times \mathcal{S} \rightarrow \mathbb{R}$ defined by

$$
\rho_{\mu}(A, B)=\mu(A \backslash B)+\mu(B \backslash A), \quad A, B \in \mathcal{S},
$$

is a finite semi-metric on $\mathcal{S}$.

Proposition 3.1. Suppose that $X$ is a Banach space and let $\mathcal{R}$ be a ring contained in $\mathcal{S}$. If $M: \mathcal{R} \rightarrow \mathcal{P}_{f}(X)$ is an additive set-valued set function such 
that $\|M(A)\| \leq \mu(A)$ for $A \in \mathcal{R}$, then $M$ is a uniformly continuous mapping from $\left(\mathcal{R}, \rho_{\mu}\right)$ into $\left(\mathcal{P}_{f}(Y), H\right)$.

Proof. For $A, B \in \mathcal{R}$ we have that

$$
\begin{aligned}
H(M(A), M(B)) & =H(M(A \backslash B)+M(A \cap B), M(B \backslash A)+M(A \cap B)) \\
& \leq H(M(A \backslash B), M(B \backslash A))+H(M(A \cap B), M(A \cap B)) \\
& \leq H(M(A \backslash B),\{0\})+H(M(B \backslash A),\{0\}) \\
& =\|M(A \backslash B)\|+\|M(B \backslash A)\| \\
& \leq \mu(A \backslash B)+\mu(B \backslash A)=\rho_{\mu}(A, B) .
\end{aligned}
$$

Proposition 3.2. Suppose that $\mathcal{R}$ is a ring dense in $\mathcal{S}$ for the topology defined by $\rho_{\mu}$ and let $X$ be a separable Banach space. If $M: \mathcal{R} \rightarrow \mathcal{P}_{k}(X)$ is an additive set-valued set function such that $\|M(A)\| \leq \mu(A)$ for all $A \in \mathcal{R}$, then $M$ can be extended to an additive set-valued set function $N: \mathcal{S} \rightarrow \mathcal{P}_{k}(X)$ such that $\|N(A)\| \leq \mu(A)$ for all $A \in \mathcal{S}$. If $\mu$ is additive, then $v(N)$ is an extension of $v(M)$.

Proof. From Theorem 2 on page 154 of [16] follows that $S_{M} \neq \emptyset$. Since $\|M(A)\| \leq \mu(A)$ for each $A \in \mathcal{R}$, we infer that each $m \in S_{M}$ is uniformly continuous on the dense class $\mathcal{R}$. By Theorem 1 on page 62 of [15] follows that each $m \in S_{M}$ can be extended to a uniformly continuous finitely additive set function $n: \mathcal{S} \rightarrow X$ such that $\|n(A)\| \leq \mu(A)$ for all $A \in \mathcal{S}$. For $A \in \mathcal{S}$, put

$$
N(A)=\overline{\{n(B) \mid B \subseteq A, B \in \mathcal{S}\}} .
$$

If $A, B \in \mathcal{S}$ with $A \cap B=\emptyset$, then

$$
\begin{aligned}
\overline{N(A)+N(B)} & =\overline{\{n(C)+n(D) \mid C \subseteq A, D \subseteq B, C, D \in \mathcal{S}\}} \\
& =\overline{\{n(C \cup D) \mid C \cup D \subseteq A \cup B\}}=N(A \cup B) ;
\end{aligned}
$$

whence $N$ is an additive set-valued set function. Clearly we have that $\|N(A)\| \leq$ $\mu(A)$ for all $A \in \mathcal{S}$. We now want to show that

$$
|d(n(A), N(A))-d(n(B), N(B))|<\|n(A)-n(B)\|+H(N(A), N(B)),
$$

because by the uniform continuity of $N$ and $n$ it will then follow that the set function $A \mapsto d(n(A), N(A))$ is uniformly continuous. Indeed, from

$$
d(n(A), N(A)) \leq d(n(B), N(A))+\|n(A)-n(B)\|,
$$

follows that we only need to prove that

$$
d(n(B), N(A)) \leq d(n(B), N(B))+H(N(A), N(B)) .
$$


For all $\epsilon>0$ we can choose $x \in N(A)$ and $y \in N(B)$ such that

$$
d(n(B), y) \leq d(n(B), N(B))+\frac{\epsilon}{2} \text { and } d(y, x) \leq d(y, N(A))+\frac{\epsilon}{2} .
$$

Consequently, for all $\epsilon>0$,

$$
\begin{aligned}
d(n(B), x) & \leq d(n(B), y)+d(y, x) \\
& \leq d(y, N(A))+d(n(B), y)+\frac{\epsilon}{2} \\
& \leq d(y, N(A))+d(n(B), N(B))+\epsilon
\end{aligned}
$$

therefore

$$
d(n(B), N(A)) \leq d(n(B), N(B))+H(N(A), N(B))+\epsilon .
$$

Since the set function $A \mapsto d(n(A), N(A))$ is identically null on the dense class $\mathcal{R}$, and since $N$ is closed-valued, we deduce that $n(A) \in N(A)$ and consequently

$$
N(A)=\overline{\left\{n(A) \mid n \in S_{N}\right\}}
$$

for all $A \in \mathcal{S}$. Also, by Proposition 2 on page III-10 of [9] we infer that $M(A)=N(A)$ for all $A \in \mathcal{R}$.

Lastly, if $\mu$ is additive, then $m$ has finite variation $v(m)$ on $\mathcal{R}, n$ has finite variation $v(n)$ on $\mathcal{S}$ and $v(n)$ is an extension of $v(m)$. Since $v(N)=v(n)$, it follows that $v(N)$ is an extension of $v(M)$.

Proposition 3.3. Suppose that $\mathcal{R}$ is a ring dense in $\mathcal{S}$ for the topology defined by $\rho_{\mu}$ and let $X$ be a separable Banach space. If $M: \mathcal{R} \rightarrow \mathcal{P}_{f b c}(X)$ is an additive set-valued set function such that $\|M(A)\| \leq \mu(A)$ for all $A \in \mathcal{R}$, then $M$ can be extended to an additive set-valued set function $N: \mathcal{S} \rightarrow \mathcal{P}_{f b}(X)$ such that $\|N(A)\| \leq \mu(A)$ for all $A \in \mathcal{S}$. If $\mu$ is additive, then $v(N)$ is an extension of $v(M)$.

Proof. By Theorem 3 on page 154 of [16] follows that there is a sequence $\left(m_{k}\right) \subseteq S_{M}$ of finitely additive set functions from $\mathcal{R}$ into $X$ such that

$$
M(A)=\overline{\left\{m_{k}(A) \mid k \in I N\right\}}
$$

for all $A \in \mathcal{R}$. Since $\|M(A)\| \leq \mu(A)$ we have that $\left\|m_{k}(A)\right\| \leq \mu(A)$ for all $A \in \mathcal{R}$ so that each $m_{k}$ is uniformly continuous on the dense class $\mathcal{R}$. For $k \in \mathbb{N}$, let $n_{k}$ denote the extension of $m_{k}$ to $\mathcal{S}$ and put

$$
N(A)=\overline{\left\{n_{k}(A) \mid k \in I N\right\}}
$$


for all $A \in \mathcal{S}$. Clearly, $N$ is an additive $\mathcal{P}_{f b c}(X)$-valued set function. Also, since $\left\|n_{k}(A)\right\| \leq \mu(A)$ for all $A \in \mathcal{S}$, we have that $\|N(A)\| \leq \mu(A)$. Lastly, since $v(N)=v\left(n_{k}\right)$ on $\mathcal{S}$ and $v(M)=v\left(m_{k}\right)$ on $\mathcal{R}$, the conclusion follows from Theorem 1 on page 62 of [15].

We now discuss the set-valued analogue of the Carathéodory-Hahn-Kluvanek theorem for additive set-valued set functions. The set-valued CarathéodoryHahn-Kluvanek theorem has been given by Kandilakis ([23], page 88, Theorem $2.6)$ for countably additive set-valued set functions. The same type of results were also obtained in [34].

First we give an example of a punctually additive set-valued set function which is not a strong multimeasure.

Example 3.4. Consider the semiring $\mathcal{R}=\{A \subseteq \mathbb{R} \mid A$ is at most countable $\}$ and define the set-valued set function $M: \mathcal{R} \rightarrow[0, \infty]$ by

$$
M(A)= \begin{cases}\{0\} & \text { if } A \text { is finite } \\ \{\infty\} & \text { if } A \text { is countable }\end{cases}
$$

To see that $M$ is punctually additive, let $A, B \in \mathcal{R}$ be such that $A \cap B=\emptyset$. If both $A$ and $B$ are finite, then $A \cup B$ is finite so that

$$
M(A \cup B)=\{0\}=M(A)+M(B) .
$$

On the other hand, if either $A$ or $B$ is countable, then $A \cup B$ is countable and

$$
M(A \cup B)=\{\infty\}=M(A)+M(B) .
$$

Finally, $M$ is not a strong multimeasure because $M\left(\bigcup_{n=1}^{\infty}\{n\}\right)=M(\mathbb{N})=$ $\{\infty\}$ and

$$
\sum_{n=1}^{\infty} M(\{n\})=\left\{y \in[0, \infty] \mid y=\sum_{n=1}^{\infty} y_{n}, y_{n} \in M(\{n\})\right\}=\{0\} .
$$

Proposition 3.5 ([2], p. 405, Theorem 1). Let $X$ be a separable Banach space and suppose that $M: \mathcal{A} \rightarrow \mathcal{P}_{f b c}(X)$ is an additive set-valued set function. If there exists a finitely additive nonnegative finite set function $\mu$ on $\mathcal{A}$ such that $M$ is $\mu$-continuous, then there exists a $\sigma$-algebra $\mathcal{S}$, a normal multimeasure $N: \mathcal{S} \rightarrow \mathcal{P}_{f b c}(X)$ and a Boolean isomorphism $i: \mathcal{A} \rightarrow \mathcal{S}$ such that $M(A)=$ $N(i(A))$ for all $A \in \mathcal{A}$. 
Proposition 3.6. Let $X$ be a Banach space and suppose that $M: \mathcal{A} \rightarrow$ $\mathcal{P}_{f b}(X)$ is a strongly additive set-valued set function such that the set function $A \rightarrow \sigma\left(x^{\prime}, M(A)\right)$ is a finitely additive measure on $\mathcal{A}$ for every $x^{\prime} \in X^{\prime}$. Then there exists a finitely additive nonnegative real-valued measure $\mu$ on $\mathcal{A}$ such that $M$ is $\mu$-continuous on $\mathcal{A}$.

Proof. By the Stone Representation Theorem there exists a compact, Hausdorff and totally disconnected topological space $\widetilde{X}$ such that $\mathcal{A}$ is isomorphic (as a Boolean algebra) with the algebra $\tilde{\mathcal{A}}$ of all clopen subsets of $\tilde{Y}$. Let $i$ be the isomorphism of $\mathcal{A}$ into $\tilde{\mathcal{A}}$. Define $\tilde{M}: \tilde{\mathcal{A}} \rightarrow \mathcal{P}_{f b}(X)$ by $\tilde{M}(i(A))=M(A)$ for all $A \in \mathcal{A}$.

Since $i(A) \rightarrow \sigma\left(x^{\prime}, \tilde{M}(i(A))\right)$ is a finitely additive measure on $\tilde{\mathcal{A}}$ for every $x^{\prime} \in X^{\prime}$, it follows that $i(A) \rightarrow \sigma\left(x^{\prime}, \tilde{M}(i(A))\right)$ is countably additive. By Proposition 2.3 on page 87 of [23] we obtain a nonnegative and real-valued countably additive measure $\tilde{\mu}$ on $\tilde{\mathcal{A}}$ such that $\tilde{M}$ is $\tilde{\mu}$-continuous on $\tilde{\mathcal{A}}$. If we define $\mu(A)=\tilde{\mu}(i(A))$ for $A \in \mathcal{A}$, then the result follows.

Proposition 3.7. Let $X$ be a Banach space and suppose that $\mathcal{S}$ is a $\sigma$-algebra of subsets of the set $T$ and let $N: \mathcal{S} \rightarrow \mathcal{P}_{w k}(X)$ be a set-valued set function such that for every $x^{\prime} \in X^{\prime}$ the set function $A \mapsto \sigma\left(x^{\prime}, N(A)\right)$ admits a Hahn decomposition. Then $N(\mathcal{S})$ is a relatively $w\left(X, X^{\prime}\right)$-compact subset of $X$.

Proof. Let $x^{\prime} \in X^{\prime}$ and let $\left(H^{+}, H^{-}\right)$be a Hahn decomposition for the signed measure $\sigma\left(x^{\prime}, N(\cdot)\right)$. Then we have that

$$
\sigma\left(x^{\prime}, \overline{N(\mathcal{S})}\right)=\sup _{A \in \mathcal{S}} \sigma\left(x^{\prime}, N(A)\right)=\sup _{A \in \mathcal{S}} \sigma\left(x^{\prime}, N\left(A \cap H^{+}\right)\right)=\sigma\left(x^{\prime}, N\left(H^{+}\right)\right) .
$$

But $N\left(H^{+}\right) \in \mathcal{P}_{w k}(X)$, so we can find an $x_{0} \in N\left(H^{+}\right)$, depending on $x^{\prime}$, such that $\sigma\left(x^{\prime}, N\left(H^{+}\right)\right)=\left(x^{\prime}, x_{0}\right)$ and hence $\sigma\left(x^{\prime}, \overline{N(\mathcal{S})}\right)=\left(x^{\prime}, x_{0}\right)$. By James' theorem we conclude that $\overline{N(\mathcal{S})}$ is a $w\left(X, X^{\prime}\right)$-compact subset of $X$.

Proposition 3.8. Let $X$ be a Banach space. If $M: \mathcal{A} \rightarrow \mathcal{P}_{f b}(X)$ is a setvalued set function such that $M(\mathcal{A})$ is a relatively weakly compact subset of $X$ and the set function $A \mapsto \sigma\left(x^{\prime}, M(A)\right)$ is a finitely additive measure on $\mathcal{A}$ for every $x^{\prime} \in X^{\prime}$, then $M$ is strongly additive.

Proof. For every $x^{\prime} \in X^{\prime}$ we have that

$$
\left|\sum_{k=1}^{n}\left(x^{\prime}, x_{k}\right)\right|=\left|\left(x^{\prime}, \sum_{k=1}^{n} x_{k}\right)\right| \leq \sum_{k=1}^{n}\left|\sigma\left(x^{\prime}, M\left(A_{k}\right)\right)\right|
$$


for all $x_{k} \in M\left(A_{k}\right)$ with $A_{k} \in \mathcal{A}, k=1,2, \ldots, n$. But since the set function $A \mapsto \sigma\left(x^{\prime}, M(A)\right)$ is of finite variation, we have that $\lim _{n \rightarrow \infty} \sum_{k=1}^{n}\left|\sigma\left(x^{\prime}, M\left(A_{k}\right)\right)\right|<\infty$.

Hence $\sum_{k=1}^{\infty} x_{k}$ is weakly unconditionally convergent and thus strongly unconditionally convergent (from Day [12]).

Summarizing the previous four results, we have:

Theorem 3.9. If $X$ is a separable Banach space and if $M: \mathcal{A} \rightarrow \mathcal{P}_{w k c}(X)$ is a set-valued set function such that the set function $\sigma\left(x^{\prime}, M(\cdot)\right)$ is a finitely additive measure on $\mathcal{A}$ for every $x^{\prime} \in X^{\prime}$, then the following are equivalent :

(a) There exist a $\sigma$-algebra $\mathcal{S}$, a multimeasure $N: \mathcal{S} \rightarrow \mathcal{P}_{w k c}(X)$ and a Boolean isomorphism $i: \mathcal{A} \rightarrow \mathcal{S}$ such that $M(A)=N(i(A))$ for all $A \in \mathcal{A}$.

(b) There exists a finitely additive nonnegative real-valued measure $\mu$ on $\mathcal{A}$ such that $M$ is $\mu$-continuous on $\mathcal{A}$.

(c) $M$ is strongly additive.

(d) $M(\mathcal{A})$ is a relatively $w\left(X, X^{\prime}\right)$-compact subset of $X$.

Proof. We only need to show that (a) implies (d).

But $M(\mathcal{A})=N(i(\mathcal{A})) \subseteq N(\mathcal{S})$, which is a relatively weakly compact subset of $X$ (by Proposition 3.7).

\section{Extension of Multimeasures and Transition Multimea- sures}

Unless otherwise stated, throughout this section we will suppose that $\mathcal{A}$ is an algebra of subsets of the set $T$ and we let $\mathcal{S}$ be the $\sigma$-algebra generated by $\mathcal{A}$.

Proposition 4.1. Let $X$ be a Banach space, suppose that the $\sigma$-algebra $\mathcal{S}$ is countably generated and let $\mu$ be a positive measure on $\mathcal{S}$. If $M: \mathcal{A} \rightarrow \mathcal{P}_{f b}(X)$ is a normal multimeasure such that $M$ is $\mu$-continuous on $\mathcal{A}$, then $M$ can be extended to a normal multimeasure $N: \mathcal{S} \rightarrow \mathcal{P}_{f b}(X)$ such that $\|N(A)\| \leq \mu(A)$ for all $A \in \mathcal{S}$.

Proof. Since $M$ is additive and the algebra $\mathcal{A}$ is countable, it follows from Proposition 2 on page III-10 of [9] that for all $A \in \mathcal{A} M(A)=\overline{\left\{m(A) \mid m \in S_{M}\right\}}$. Furthermore, by Theorem 2 on page 59 of [21], it follows that $\lim _{\mu(A) \rightarrow 0}\|M(A)\|=$ 0 so that $\lim _{\mu(A) \rightarrow 0}\|m(A)\|=0$ for all $m \in S_{M}$ and $A \in \mathcal{A}$. 
Consequently, each $m \in S_{M}$ is uniformly continuous on the dense class $\mathcal{A}$ and thus may be extended to a uniformly continuous set function $n: \mathcal{S} \rightarrow X$. If, for all $A \in \mathcal{S}$, we put $N(A)=\overline{\{n(B) \mid B \subseteq A, B \in \mathcal{S}\}}$, then again we can prove that $N(A)=\overline{\left\{n(A) \mid n \in S_{N}\right\}}$ for $A \in \mathcal{S}$. Clearly $N$ extends $M$ to $\mathcal{S}$.

It only remains to show that $N$ is a normal multimeasure. Put $N^{\prime}(A)=$ $\{n(B) \mid B \subseteq A, B \in \mathcal{S}\}$ and let $\left(A_{k}\right)$ be a sequence of mutually disjoint sets in $\mathcal{S}$. Then

$$
\begin{aligned}
& H\left(N^{\prime}\left(\bigcup_{k=1}^{\infty} A_{k}\right), \sum_{k=1}^{\infty} N^{\prime}\left(A_{k}\right)\right) \\
= & H\left(\sum_{k=1}^{n} N^{\prime}\left(A_{k}\right)+N^{\prime}\left(\bigcup_{k=n+1}^{\infty} A_{k}\right), \sum_{k=1}^{n} N^{\prime}\left(A_{k}\right)+\sum_{k=n+1}^{\infty} N^{\prime}\left(A_{k}\right)\right) \\
\leq & H\left(N^{\prime}\left(\bigcup_{k=n+1}^{\infty} A_{i}\right), \sum_{k=n+1}^{\infty} N^{\prime}\left(A_{k}\right)\right) \\
\leq & \left\|N^{\prime}\left(\bigcup_{k=n+1}^{\infty} A_{k}\right)\right\|+\left\|\sum_{k=n+1}^{\infty} N^{\prime}\left(A_{k}\right)\right\| \leq 2 \sum_{k=n+1}^{\infty} v\left(N^{\prime}, A_{k}\right) \longrightarrow 0
\end{aligned}
$$

as $n \rightarrow \infty$. This shows that $N^{\prime}$ is a strong multimeasure. By Proposition 5 on page 57 of [21] it follows that $N$ is a $\mathcal{P}_{f b}(X)$-valued normal multimeasure. Lastly, since $\|n(A)\| \leq \mu(A)$ for all $A \in \mathcal{S}$, we conclude that $\|N(A)\| \leq$ $\mu(A)$.

Proposition 4.2. Let $X$ be a separable Banach space and let $\mu$ be a positive measure on $\mathcal{S}$. If $M: \mathcal{A} \rightarrow \mathcal{P}_{k c}(X)$ is a $\mu$-continuous normal multimeasure, then $M$ can be extended to a normal multimeasure $N: \mathcal{S} \rightarrow \mathcal{P}_{k c}(X)$ such that $\|N(A)\| \leq \mu(A)$ for every $A \in \mathcal{S}$.

Proof. Since $M(A) \in \mathcal{P}_{k c}(X)$ for all $A \in \mathcal{A}$ and $X$ is separable, there is a countable set $\left\{x_{1}, x_{2}, \ldots\right\}$ which is dense in $M(A)$. By Theorem 3 on page 154 of [16] there exists a sequence $\left(m_{k}\right) \subseteq S_{M}$ such that $m_{k}(A)=x_{k}$ for all $A \in \mathcal{A}$ and by the convexity of $M$ we have that $M(A)=\overline{c o}\left\{m_{k}(A) \mid k \in \mathbb{N}\right\}$ for all $A \in \mathcal{S}$. Let $n_{k}$ be the extension of $m_{k}$ to $\mathcal{S}$ and for every $A \in \mathcal{S}$ put

$$
N(A)=\overline{c o}\left\{n_{k}(A) \mid k \in \mathbb{N}\right\}
$$

If we put $N^{\prime}(A)=\left\{n_{k}(A) \mid k \in \mathbb{N}\right\}$, then by Proposition 5 on page 57 of [21] we only need to show that $N^{\prime}$ is a strong multimeasure. Let $\left(A_{k}\right)$ be a sequence 
of mutually disjoint sets in $\mathcal{S}$ and let $A=\cup_{k=1}^{\infty} A_{k}$. Then

$$
\begin{aligned}
H\left(N^{\prime}(A), \sum_{k=1}^{\infty} N^{\prime}\left(A_{k}\right)\right) & \leq H\left(N^{\prime}(A), \sum_{k=1}^{n} N^{\prime}\left(A_{k}\right)\right)+\sum_{k=n+1}^{\infty}\left\|N^{\prime}\left(A_{k}\right)\right\| \\
& =H\left(N^{\prime}(A), N^{\prime}\left(\bigcup_{k=1}^{n} A_{k}\right)\right)+\sum_{k=n+1}^{\infty} v\left(N^{\prime}, A_{k}\right) \longrightarrow 0
\end{aligned}
$$

as $n \rightarrow \infty$. Hence $N^{\prime}(A)=\sum_{k=1}^{\infty} N^{\prime}\left(A_{k}\right)$ and therefore $N^{\prime}$ is a strong multimeasure.

For the rest of this section we suppose that $(\Omega, \mathcal{T})$ is a complete measurable space and $\mathcal{R}$ is a ring of subsets of $T$. Let $\mathcal{S}$ be the $\sigma$-ring generated by $\mathcal{R}$ and let $\lambda: \Omega \times \mathcal{S} \rightarrow \mathbb{R}_{+}$be a transition measure.

Theorem 4.3. If $M: \Omega \times \mathcal{R} \rightarrow \mathcal{P}_{k c}\left(\mathbb{R}^{n}\right)$ is a strong transition multimeasure of bounded variation such that $\lim _{\lambda(\omega, A) \rightarrow 0} M(\omega, A)=0$ for $(\omega, A) \in(\Omega, \mathcal{R})$, then $M$ can be extended to a strong transition multimeasure $N: \Omega \times \mathcal{S} \rightarrow \mathcal{P}_{k c}\left(\mathbb{R}^{n}\right)$ of bounded variation such that $\lim _{\lambda(\omega, A) \rightarrow 0} N(\omega, A)=0$ for $(\omega, A) \in(\Omega, \mathcal{S})$.

Proof. For all $A \in \mathcal{R}$, define $F(\omega)=M(\omega, A)$ for $\omega \in \Omega$. Since $F$ is a measurable multifunction, there is a sequence $\left(f_{k}\right) \subseteq S_{F}$ of measurable functions $f_{k}: \Omega \rightarrow \mathbb{R}^{n}$ such that $F(\omega)=\overline{\left\{f_{k}(\omega) \mid k \in \mathbb{N}\right\}}$ for all $\omega \in \Omega$ (see [25], page 69, Theorem 7.8). By Theorem 2 on page 92 of [21] follows that there is a sequence $\left(m_{k}\right) \subseteq T S_{M}$ such that $f_{k}(\omega)=m_{k}(\omega, A)$ for all $\omega \in \Omega$, and by the convexity of $M$ follows that

$$
M(\omega, A)=\overline{c o}\left\{m_{k}(\omega, A) \mid k \in \mathbb{N}\right\}
$$

for every $(\omega, A) \in \Omega \times \mathcal{R}$. Furthermore, $\lim _{\lambda(\omega, A) \rightarrow 0} M(\omega, A)=0$ so that $\lim _{\lambda(\omega, A) \rightarrow 0} m_{k}(\omega, A)=0$ for all $(\omega, A) \in \Omega \times \mathcal{R}$. By Proposition 2 on page 96 of [21] we may extend each $m_{k}$ to a unique transition measure $n_{k}: \Omega \times \mathcal{S} \rightarrow \mathbb{R}^{n}$. For all $(\omega, A) \in \Omega \times \mathcal{S}$ put $N(\omega, A)=\overline{c o}\left\{n_{k}(\omega, A) \mid k \in \mathbb{N}\right\}$. Since, for all $A \in \mathcal{S}$, we have that $\omega \mapsto\left\{n_{k}(\omega, A) \mid k \in \mathbb{N}\right\}$ is a measurable multifunction, it follows that $N$ is also a measurable multifunction. Clearly $A \mapsto N(\omega, A)$ is a $\mathcal{P}_{k c}\left(\mathbb{R}^{n}\right)$ valued strong multimeasure.

Theorem 4.4. Let $X$ be a Hausdorff locally convex real vector space and $X^{\prime}$ a separable Fréchet space. If $M: \Omega \times \mathcal{R} \rightarrow \mathcal{P}_{k c}(X)$ is a weak transition multimeasure of bounded variation such that $\lim _{\lambda(\omega, A) \rightarrow 0} M(\omega, A)=0$ for $(\omega, A) \in(\Omega, \mathcal{R})$, then $M$ can be extended to a weak transition multimeasure $N: \Omega \times \mathcal{S} \rightarrow \mathcal{P}_{k c}(X)$ of bounded variation such that $\lim _{\lambda(\omega, A) \rightarrow 0} N(\omega, A)=0$ for $(\omega, A) \in(\Omega, \mathcal{S})$. 
Proof. If, for all $A \in \mathcal{R}$, we define $F(\omega)=M(\omega, A)$, then $F$ is a measurable multifunction. Since $(\Omega, \mathcal{T})$ is complete, it is a Souslin family. Hence (see Theorem 8.4 of [32]), there is a sequence $\left(f_{k}\right) \subseteq S_{\text {extF }}$ of measurable functions $f_{k}: \Omega \rightarrow X$ such that $F(\omega)=\overline{c o}\left\{f_{k}(\omega) \mid k \in \mathbb{N}\right\}$. By Proposition 1 on page 95 of [21] there is a sequence $\left(m_{k}\right) \subseteq T S_{M}$ such that $f_{k}(\omega)=m_{k}(\omega, A)$ for all $\omega \in \Omega$, and hence $M(\omega, A)=\overline{c o}\left\{m_{k}(\omega, A) \mid k \in \mathbb{N}\right\}$ for every $(\omega, A) \in \Omega \times \mathcal{R}$. Again, as before, $\lim _{\lambda(\omega, A) \rightarrow 0} M(\omega, A)=0$ so that $\lim _{\lambda(\omega, A) \rightarrow 0} m_{k}(\omega, A)=0$ for all $(\omega, A) \in \Omega \times \mathcal{R}$. By Proposition 2 on page 96 of [21] we may extend each $m_{k}$ to a unique transition measure $n_{k}: \Omega \times \mathcal{S} \rightarrow X$.

For all $(\omega, A) \in \Omega \times \mathcal{S}$ put

$$
N(\omega, A)=\overline{c o}\left\{n_{k}(\omega, A) \mid k \in \mathbb{N}\right\} .
$$

Since $A \mapsto N(\omega, A)$ is a strong multimeasure, it follows from Theorem 5.1 of [27] that $A \mapsto N(\omega, A)$ is a weak multimeasure.

\section{References}

[1] R. Aló, A de Korvin, \& C. Roberts, p-integrable selectors of multimeasures, Int. J. Math. Math. Sci., 2 (1979), 209-221.

[2] R. Aló, A de Korvin, \& C. Roberts, On some properties of continuous multimeasures, J. Math. Anal. Appl., 75 (1980), 402-410.

[3] Z. Artstein, Set-valued measures, Trans. Amer. Math. Soc., 65 (1972), $103-125$.

[4] L. Blume, New techniques for the study of stochastic equilibrium processes, J. Math. Econom., 9 (1982), 61-70.

[5] C. Castaing, Le théorème de Dunford-Pettis généralisé, C. R. Acad. Sci., 238 (1969), 327-329.

[6] A. Costé, Sur l'integration par rapport à une multimesure de Radon, C. R. Acad. Sci., 278 (1974), 545-548.

[7] A. Costé, Sur les multimesures à valeurs fermées bornées d'un espace de Banach, C. R. Acad. Sci., 280 (1975), 567-570.

[8] A. Costé, La propriétè de Radon-Nikodým en intégration multivoque, C. R. Acad. Sci., 280 (1975), 1515-1518.

[9] A. Costé, Contribution à la théorie de l'intégration multivoque, Ph.D. Thesis, University of Paris, 1977. 
[10] A. Costé \& R. Pallu de la Barrière, Sur l'ensemble des sections d'une multimesures à valeurs convexes, C. R. Acad. Sci., 282 (1976), 829-832.

[11] A. Costé \& R. Pallu de la Barrière, Un théorème de Radon-Nikodým pour les multimesures à valeurs convexes fermées localement compactes sans droit, C. R. Acad. Sci., 280 (1975), 255-258.

[12] M. M. Day, Normed spaces, Springer-Verlag, Berlin, 1972.

[13] G. Debreu \& D. Schmeidler, The Radon-Nikodým derivative of a correspondence, in: Proc. Sixth Berkeley Sympos. Math. Statist. and Probability (Berkeley, Calif., 1970/1971), vol. II: Contributions to Probability Theory, Part 2, 41-56, Univ. California Press, Berkeley, Calif., 1972.

[14] J. Diestel, \& J. J. Uhl jr, Vector measures, Math. Surveys. Vol.15, Amer. Math. Soc., Providence, R.I, 1977.

[15] N. Dinculeanu, Vector measures, Pergamon, New York, 1967.

[16] C. Godet-Thobie, Sur les sélections de mesures géneralisées, C. R. Acad. Sci., 271 (1970), 153-156.

[17] C. Godet-Thobie, Sur les intégrales de fonctions réelles par rapport à une mesure géneralisées et ses sélections, C. R. Acad. Sci., 271 (1970), 497-500.

[18] C. Godet-Thobie, Sur les multimesures de transition, C. R. Acad. Sci., 278 (1974), 1367-1369.

[19] C. Godet-Thobie, Some results about multimeasures and their selectors, in: Measure Theory (Oberwolfach, 1979), edited by D. Kölzow, Lecture Notes in Mathematics No. 794, 112-116, Springer-Verlag, Berlin, 1980.

[20] C. Godet-Thobie, Sélections de multimesures. Applications à un théorème de Radon-Nikodým multivoque, C. R. Acad. Sci., 279 (1974), 603-606.

[21] C. Godet-Thobie, Multimesures et multimesures de transition, Ph.D thesis, University of Paris, 1975.

[22] F. Hiai, Radon-Nikodým theorems for set-valued measures, J. Multivariate Anal., 8 (1978), 96-118.

[23] D. A. Kandilakis, On the extension of multimeasures and integration with respect to a multimeasure, Proc. Amer. Math. Soc., 116 (1992), 85-92. 
[24] I. Kluvanek, The extension and closure of a vector measure, In: Vector and operator valued measures and applications, in: Proc. Sympos. (Snowbird Resort, Alta, Utah, 1972), 175-190, Academic Press, New York, 1984.

[25] P. Maritz, Integration of set-valued functions, Ph.D. thesis, University of Leiden, 1975.

[26] N. S. Papageorgiou, On the theory of Banach space valued multifunctions, Part 1: Integration and conditional expectation, J. Multivariate Anal., 17 (1985), 185-206.

[27] N. S. Papageorgiou, On the theory of Banach space valued multifunctions, Part 2: Set valued martingales and set valued measures, J. Multivariate Anal., 17 (1985), 207-227.

[28] N. S. Papageorgiou, On the properties of multimeasures with values in a Banach space, Annales Societatis Masthematicae Polonae, Series I: Commentationes Mathematicae, XXXII (1992), 111-121.

[29] N. S. Papageorgiou, Radon-Nikodým theorems for multimeasures and transition multimeasures, Proc. Amer. Math. Soc., 111 (1991), 465-474.

[30] D. Schmeidler, Convexity and compactness in countably additive correspondences, in: Differential games and related topics, North Holland, Amsterdam, 1971.

[31] K. Vind, Edgeworth allocations in an exchange economy with many traders, Internat. Econom. Rev., 5 (1964), 165-177.

[32] D. Wagner, Survey of measurable selection theorems, SIAM J. Control Optim., 15 (1977), 859-903.

[33] Z. Wenxiu, M. Jifeng \& L. Aijie, The extension of a compact set-valued measure, J. Math. Research. Exp., 10 (1990), 35-42.

[34] X. Xiaoping, C. Lixin, L. Goucheng \& Y. Xiaobo, Set valued measures and integral representation, Comment. Math. Univ. Carolinae., 37 (1996), 269-284. 
H. E. BRink AND P. MARITZ 